\title{
Case Report \\ Thrombotic Occlusion of All Left Coronary Branches in a Young Woman with Severe Ulcerative Colitis
}

\section{Carl Gunnar Gustavsson, ${ }^{1}$ Peter J. Svensson, ${ }^{2}$ Erik Hertervig, ${ }^{3}$ Lennart Sandhall, ${ }^{4}$ Peter Hårdhammar, ${ }^{5}$ Natascia Malcevschi-Lind, ${ }^{6}$ and Sven-Erik Olsson ${ }^{7}$}

\author{
${ }^{1}$ Department of Cardiology, Skane University Hospital Malmoe, 20502 Malmoe, Sweden \\ ${ }^{2}$ Department for Coagulation Disorders, Skane University Hospital Malmoe, 20502 Malmoe, Sweden \\ ${ }^{3}$ Department of Gastroenterology, Skane University Hospital Lund, 22185 Lund, Sweden \\ ${ }^{4}$ Department of Radiology, Helsingborg Hospital, 25187 Helsingborg, Sweden \\ ${ }^{5}$ Department of Internal Medicine, Central Hospital, Halmstad Hospital, 30185 Halmstad, Sweden \\ ${ }^{6}$ Private Practice, Upplandsgatan 16, 21429 Malmoe, Sweden \\ ${ }^{7}$ Department of Internal Medicine, Helsingborg Hospital, 25187 Helsingborg, Sweden
}

Correspondence should be addressed to Carl Gunnar Gustavsson, CG@gustavsson.se

Received 28 February 2011; Accepted 7 April 2011

Academic Editors: J. Morais, I. Ott, and F. Perticone

Copyright (c) 2011 Carl Gunnar Gustavsson et al. This is an open access article distributed under the Creative Commons Attribution License, which permits unrestricted use, distribution, and reproduction in any medium, provided the original work is properly cited.

\begin{abstract}
Background. The thrombosis risk is increased in active ulcerative colitis. The limited number of reported complications have predominantly been cerebrovascular but other vessel territories may also be affected. Patient. During a severe attack of ulcerative colitis a 37-year-old woman suffered occlusion of all left coronary artery branches. Serial angiographies showed progressive recanalisation of the coronary arteries during anticoagulation, but no atherosclerotic stenosis. The cause of infarction was thus considered to be an extensive coronary thrombosis. However, a large battery of blood tests failed to identify any procoagulant abnormality. Conclusion. Evidence is now accumulating that the increased thrombosis risk also may involve the coronary arteries, even in young patients. To the best of our knowledge this is the third reported case of myocardial infarction despite angiographically normal coronary arteries in a patient with active ulcerative colitis. The extent of affected myocardium was in this case exceptionally large.
\end{abstract}

Patients with inflammatory bowel disease have an increased risk of thrombotic complications. The affected patients are usually relatively young; in one publication, the mean age of the 30 patients with ulcerative colitis and cerebrovascular complications was 28.6 years [1]. Thrombosis of arm or leg arteries has in some cases even resulted in amputation.

We report the thrombotic occlusion of all left coronary branches in a 37-year-old woman with active ulcerative colitis. She had no family history of inflammatory bowel disease, coronary heart disease, or thromboembolism. Since her teens, she had had periods of abdominal pain and occasionally noted blood-stained stools. At the age of 35 , she stopped smoking and a few months later, she had an episode of obvious proctitis.
Six months later, proctitis reoccurred. The condition worsened despite steroid enemas and oral steroids, and she was, therefore, hospitalized for treatment with intravenous steroids and total parenteral nutrition. After one week of bowel rest, she still had 8-10 bloody stools per day. At this point, she felt a pain radiating from her right arm across the chest. ECG showed myocardial ischemia but no obvious infarction.

The first coronary angiography was performed about 15 hours after onset of chest pain. The left main stem and the most proximal parts of the left anterior descending branch (LAD), an intermediate branch, and the circumflex branch were all angiographically normal, but there was no contrast flow at all to the more distal parts of any of these three branches (Figure 1). The right coronary artery was not 


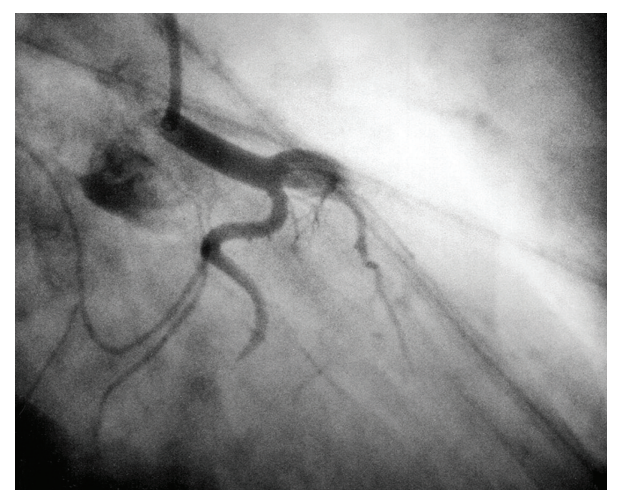

FIgURE 1: At the first angiogram all large branches of the left coronary artery were occluded. Right anterior oblique (RAO) projection.

catheterized selectively, but it appeared wide open with a rapid flow when contrast was injected into the aortic root.

A guide wire could easily be passed to the LAD periphery, but we were not able to achieve reperfusion. Aspiration catheters were not available at that time. About 15 hours had then elapsed from the onset of chest pain, and there was an extensive Q wave infarction on ECG. Thrombolytic agents and platelet glucoprotein IIb/IIIa receptor antagonists were considered contraindicated due to the severely active colitis with frequent blood-stained stools. The patient developed a transmural myocardial infarction with a CK-B maximum of $3.90 \mathrm{~kat} / \mathrm{l}($ normal $<0.08 \mathrm{~kat} / \mathrm{l})$.

She was started on cyclosporine $4 \mathrm{mg} / \mathrm{kg}$ and received heparin as an anticoagulant, with an additional expectation that her colitis would benefit from this. However, she continued to have frequent blood-stained watery stools between $1200-1500 \mathrm{~mL}$ daily in spite of total parenteral nutrition, cyclosporine, high-dose intravenous steroids, and heparin. There was a need for emergent colectomy which was successfully performed two weeks after the myocardial infarction when her cardiac situation was considered stable enough.

Repeat coronary angiography was performed twice. Twelve days after admission, before the colectomy, the circumflex artery was open without any stenosis, but the left anterior descending artery was occluded in its mid portion with a partly wall-adherent thrombus proximal to the occlusion, and the intermediate branch was subtotally occluded (Figure 2). After treatment with warfarin, a third coronary angiography was performed 3 months after the infarction with the intention to dilate the LAD, since the patient had had some angina pectoris. However, in the meantime, she had become free of her chest discomfort, and all left coronary artery branches were now open with only minor irregularities remaining (Figure 3 ).

Seven years later, an echocardiographic examination showed a left ventricular end-diastolic diameter of $68 \mathrm{~mm}$, and the ejection fraction was $30 \%-35 \%$, that is, the left ventricle was moderately dilated with a moderately reduced systolic function. Her functional capacity was slightly reduced

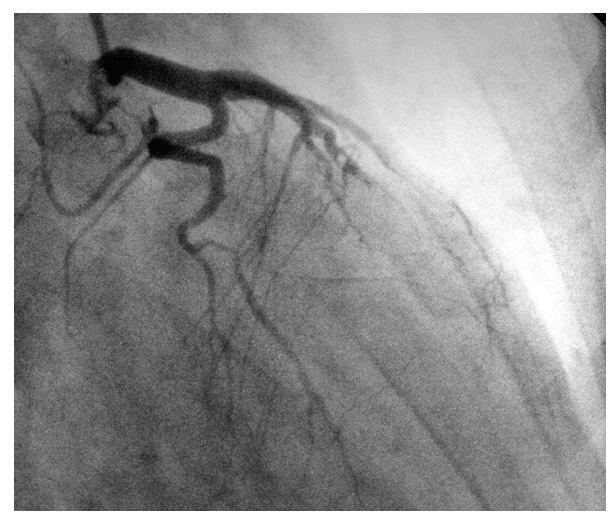

FIGURE 2: The first repeat angiogram twelve days after infarction showed an open circumflex artery, but the LAD was occluded distally with a wall adherent thrombus proximal to the occlusion. Right anterior oblique (RAO) projection.

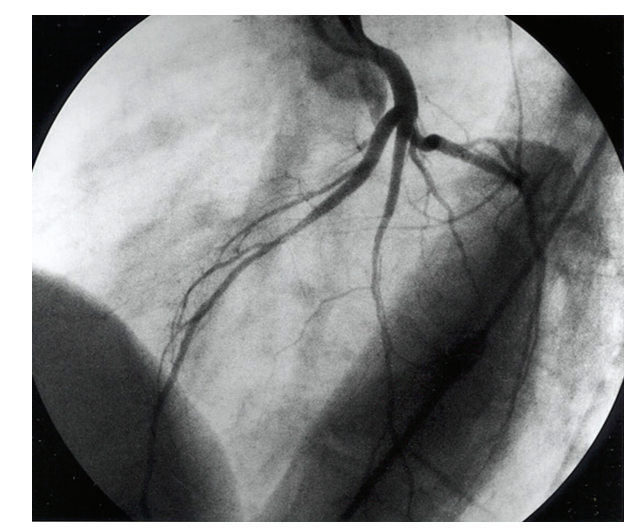

FIGURE 3: Three months after the infarction, only minor irregularities in the distal part of the LAD remained. Left anterior oblique (LAO) projection.

to NYHA class 2, and she was treated with ramipril $3.75 \mathrm{mg}$ b.i.d., bisoprolol $2.5 \mathrm{mg}$ daily, warfarin, and simvastatin.

We think the occlusions of the coronary arteries in this patient were purely thrombotic, since serial angiography showed progressive resolution of the occlusions with only minor and hemodynamically nonsignificant irregularities remaining at the latest study. The wall-adherent thrombus in the mid portion of the partially reopened LAD on the second angiography is in line with this interpretation.

To the best of our knowledge, there are only two previously reported cases of thrombotic occlusion of angiographically normal coronary arteries complicating active ulcerative colitis $[2,3]$. The present case differs by the exceptionally large infarction extent with occlusion of all left coronary branches. Contrary to the other two cases, we also showed progressive resolution from occlusion of all vessel branches to open vessels without significant stenosis and with walladherent thrombus visualized on the second angiography. In the other two cases, angiography was performed after partial or full lysis of the thrombus [2,3]. Excluding postoperative myocardial infarctions, we found three more previously 
reported cases of coronary complications in inflammatory bowel disease $[4,5]$. However, those patients were older than 60 years, and at least two of them had multiple coronary risk factors. Without angiography, it, therefore, cannot be ruled out that they had some degree of atherosclerotic coronary artery disease.

Several procoagulant abnormalities have been reported in patients with inflammatory bowel diseases, but the findings have been inconsistent. Despite a large battery of coagulation tests we could not to identify any distinct procoagulant abnormality, inherited or acquired. Activated partial thromboplastin time (APTT), dilute Russel's venom viper time (dRVVT), concentrations of antithrombin, protein C, and plasminogen as well as tests for factor $\mathrm{V}$ mutation or prothrombin mutation were all normal. The free fraction of protein $S$ was slightly reduced but with a normal total concentration and prothrombin complex (INR) 1.6 due to warfarin therapy. The only possibly procoagulant marker found was blood group A, which has been associated with an increased thrombosis risk due to an increased plasma level of von Willebrandt Factor and Factor VIII compared to blood group 0 .

The absence of such abnormalities in our patient does not preclude the interpretation as a thrombotic event. Vascular injury including endothelial cells damage could have contributed to this [6] as well as general prothrombotic alterations associated with inflammatory activity such as thrombocytosis, increased levels of factor VIII and von Willebrand factor, hypoalbuminemia, hyperfibrinogenemia, and dehydration.

\section{References}

[1] D. R. Johns, "Cerebrovascular complications of inflammatory bowel disease," American Journal of Gastroenterology, vol. 86, no. 3, pp. 367-370, 1991.

[2] M. Efremidis, E. Prappa, and F. Kardaras, "Acute myocardial infarction in a young patient during an exacerbation of ulcerative colitis," International Journal of Cardiology, vol. 70, no. 2, pp. 211-212, 1999.

[3] B. Mutlu, C. M. H. Ermeydan, F. Enç et al., "Acute myocardial infarction in a young woman with severe ulcerative colitis," International Journal of Cardiology, vol. 83, no. 2, pp. 183-185, 2002.

[4] M. J. Webberley, M. T. Hart, and V. Melikian, "Thromboembolism in inflammatory bowel disease: role of platelets," Gut, vol. 34, no. 2, pp. 247-251, 1993.

[5] R. W. Talbot, J. Heppell, R. R. Dozois, and R. W. Beart, "Vascular complications of inflammatory bowel disease," Mayo Clinic Proceedings, vol. 61, no. 2, pp. 140-145, 1986.

[6] J. C. Souto, E. Martinez, M. Roca et al., "Prothrombotic state and signs of endothelial lesion in plasma of patients with inflammatory bowel disease," Digestive Diseases and Sciences, vol. 40, no. 9, pp. 1883-1889, 1995. 


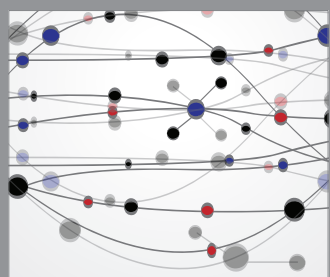

The Scientific World Journal
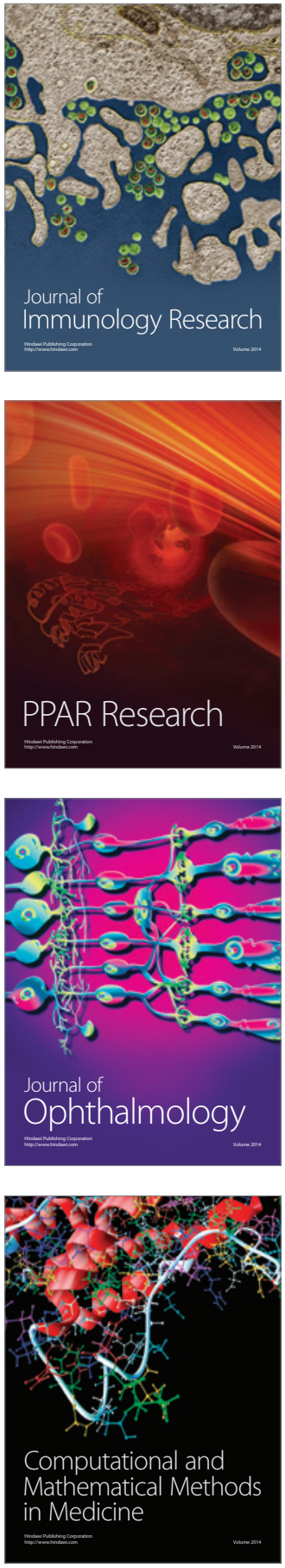

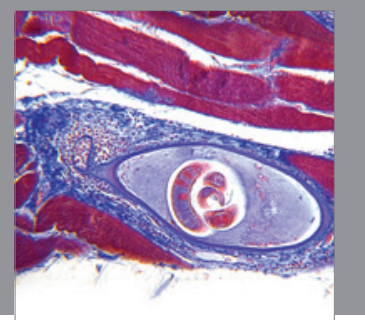

Gastroenterology

Research and Practice
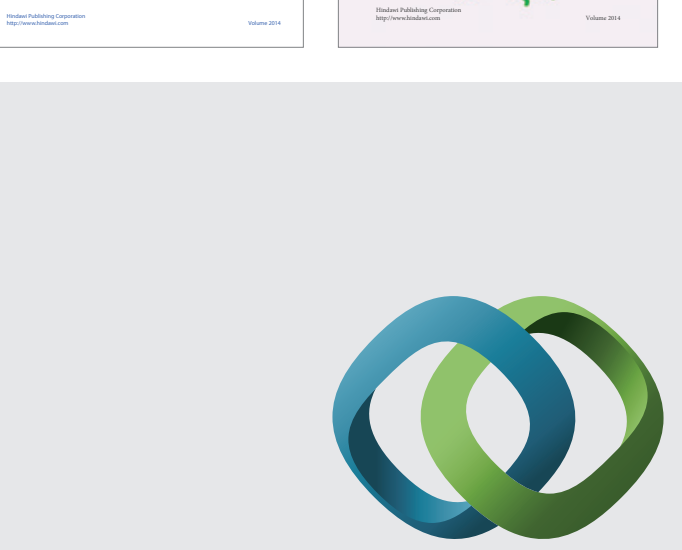

\section{Hindawi}

Submit your manuscripts at

http://www.hindawi.com
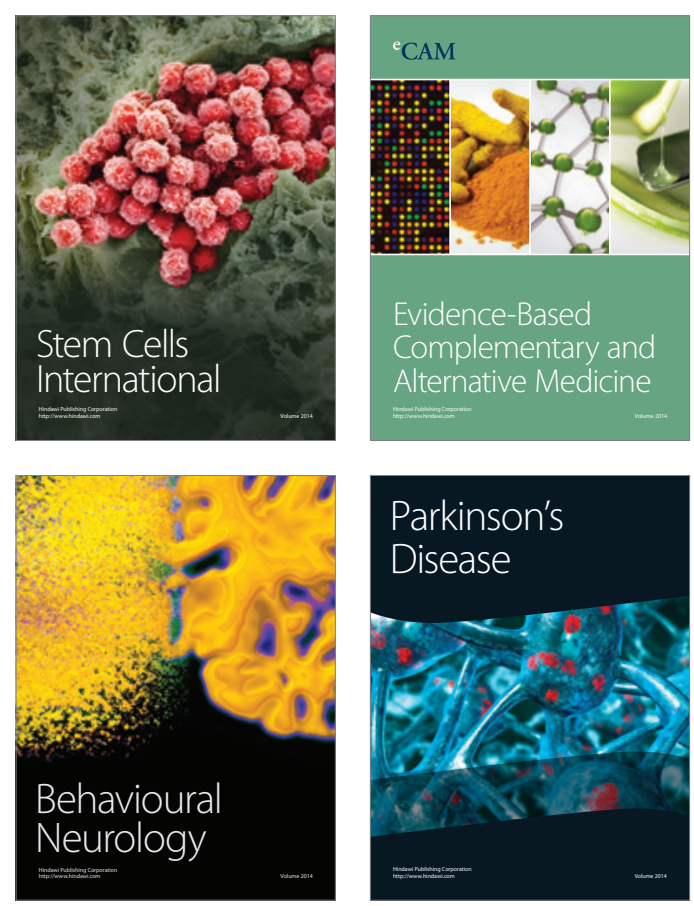

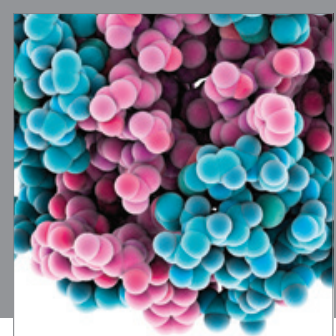

Journal of
Diabetes Research

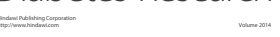

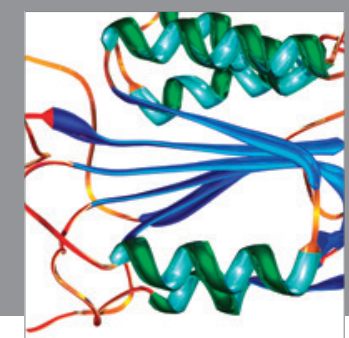

Disease Markers
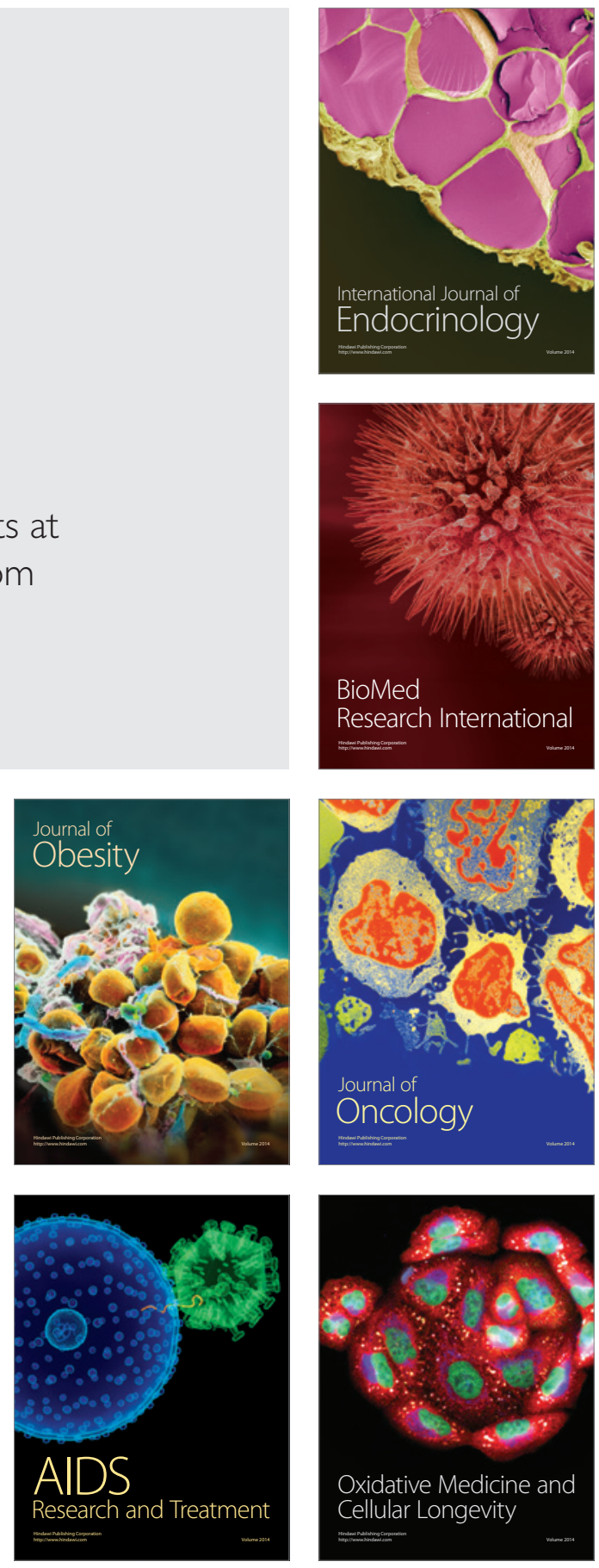